\title{
Unification of a bounce with a viable dark energy era in Gauss-Bonnet gravity
}

\author{
S. D. Odintsov, ${ }^{1,2, *}$ V. K. Oikonomou $\oplus^{3,4, \dagger}$ F. P. Fronimos $\oplus^{3, \ddagger}$ and K. V. Fasoulakos $\oplus^{3, \S}$ \\ ${ }^{1}$ ICREA, Passeig Luis Companys, 23, 08010 Barcelona, Spain \\ ${ }^{2}$ Institute of Space Sciences (IEEC-CSIC) C. Can Magrans s/n, 08193 Barcelona, Spain \\ ${ }^{3}$ Department of Physics, Aristotle University of Thessaloniki, Thessaloniki 54124, Greece \\ ${ }^{4}$ Laboratory for Theoretical Cosmology, Tomsk State University of Control Systems and Radioelectronics, \\ 634050 Tomsk, Russia (TUSUR)
}

(Received 11 September 2020; accepted 23 October 2020; published 16 November 2020)

\begin{abstract}
In this work we demonstrate that it is possible to describe a primordial bounce with the dark energy era in a unified way, in the context of Gauss-Bonnet modified gravity. In particular, the early-time bounce has a nearly scale-invariant power spectrum of primordial scalar curvature perturbations, while the dark energy era is a viable one, meaning that it mimics the $\Lambda$ cold dark matter model and is also compatible with the Planck 2018 data on cosmological parameters. In addition, our analysis indicates that the dark energy era is free from dark energy oscillations, which occur in the context of $f(R)$ gravity. We further address the latter issue by examining $f(R)$ extensions of Gauss-Bonnet models, and we show that the $f(R)$ gravity part of the action actually produces the dark energy oscillations at redshifts $z \sim 4$.
\end{abstract}

DOI: $10.1103 /$ PhysRevD.102.104042

\section{INTRODUCTION}

The dark sector of the Universe constitutes the most mysterious problems in theoretical physics and cosmology, since these two sectors control the evolution of up to $96 \%$ of the Universe. The dark sector consists of two partsdark matter and dark energy - both of which still lack a consistent explanation. With regard to dark matter, it is still unknown whether it is controlled by a weakly interacting massive particle [1-6] or if it is simply some manifestation of a modified version of general relativity [7]. On the other hand, dark energy refers to the late-time acceleration of the Universe (first observed in the late 1990s [8]), and this mysterious dark energy era has attracted a lot of attention in the literature [9-22]. Out of all of the theoretical approaches to consistently describe the dark energy era, modified gravity is to date the most promising description; see, for example, the reviews [23-28].

Apart from the mysterious dark sector of the Universe, another major issue (which will hopefully be explained in the next two decades) is the primordial post-quantum gravity era of our Universe. To date there are two candidate descriptions for this primordial era: the inflationary scenario [29-31] and the bouncing cosmology scenario [3240]. Both descriptions produce a nearly scale-invariant power spectrum for the primordial scalar curvature

\footnotetext{
*odintsov@ice.cat

†.k.oikonomou1979@gmail.com

fotisfronimos@gmail.com

${ }^{\S}$ kvfasoulakos@gmail.com
}

perturbations, with the cosmological bounces having the attribute of also producing a cosmological evolution free from the initial singularity.

In the context of modified gravity it is often possible to describe the early- and late-time eras of our Universe in a unified way; see, for example, Refs. [41,42]. In fact, modified gravity might serve as the only consistent description of dark energy beyond general relativity. The reason for this is simple, since general relativity can describe late-time acceleration in a restricted way by using a scalar field which produces either quintessential or phantom evolution, and also a simple cosmological constant may describe a de Sitter evolution at late times. But phantom scalar fields are not necessarily the best description for the late-time era, since these inevitably drive the Universe towards a big rip singularity [43], and also phantom fields can be sources of instabilities. Modified gravity can successfully provide a consistent late-time era that can mimic a quintessential, de Sitter, or even a phantom dark energy era; see, for example, the reviews [23-28] for more details on these issues.

One promising sector of modified gravity theories is Gauss-Bonnet gravity [44-59], in which the Gauss-Bonnet invariant appears in the Lagrangian in a nonlinear way. Also, extensions of general relativity that contain higher orders of the Riemann and Ricci tensors can be found in Refs. [60-62]. The general focus of this paper is on $f(R, \mathcal{G})$ theories of gravity [48,59,63-65]; specifically, we mainly focus on theories of the form $R+f(\mathcal{G})$, in order to avoid having primordial superluminal perturbation modes, but for reasons 
that will be explained shortly we also study the late-time behavior of an $f(R)+g(\mathcal{G})$ model. Our aim is twofold: to find an appropriate model of Gauss-Bonnet gravity that can describe the dark energy era in a consistent way, and to demonstrate that in some Gauss-Bonnet models it is possible to provide a unified description of the primordial postquantum era and the dark energy era with the same model. One of the models that we present in this paper is capable of describing a primordial type IV singular bounce and a latetime dark energy era, which mimics the $\Lambda$ cold dark matter $(\Lambda \mathrm{CDM})$ model and produces values for the cosmological quantities of interest that are compatible with the Planck 2018 data on cosmological parameters [66]. With regard to the primordial singular bounce, type IV singularities are smooth and thus do not affect the evolution of the Universe in an extreme way (as the crushing types of singularities do). In addition, it was shown in an earlier work that this particular singular bounce generates a nearly scale-invariant power spectrum of the primordial scalar curvature perturbations, compatible with the latest Planck 2018 constraints on inflation. Apart from the fact that our Gauss-Bonnet model of the form $R+f(\mathcal{G})$ can produce both a primordial singular bounce and a dark energy era compatible with the $\Lambda \mathrm{CDM}$ model and the latest Planck observations [66], one major outcome of our work is that this specific type of models produce a dark energy era free from dark energy oscillations, known to be present in $f(R)$ gravity models at large redshifts $z \sim 4$. In fact, in order to verify this issue we also examine the late-time phenomenology of a model of the form $f(R)+$ $g(\mathcal{G})$ and, as we demonstrate, this type of models can also be compatible with both the Planck 2018 observations and the $\Lambda \mathrm{CDM}$ model, but it is not free from dark energy oscillations. Thus, our work indicates the fact that the dark energy oscillations are possibly due to the $f(R)$ gravity sector.

\section{MODIFYING THE GAUSS-BONNET GRAVITY THEORETICAL FRAMEWORK TO STUDY THE DARK ENERGY ERA}

As we already mentioned, in this work we focus on the unification of a singular bounce with the dark energy era, and to our knowledge this is the first time that this proposal has been considered quantitatively. In this section we present the theoretical framework of a general $f(R, \mathcal{G})$ gravity and appropriately modify the Friedmann equation by using appropriate statefinder quantities, in order to study the late-time era in an optimal way. The starting point of our work is obviously the gravitational action, and we assume an $f(R, \mathcal{G})$ model accompanied by the presence of perfect matter fluids, with the following gravitational action:

$$
S=\int d^{4} x \sqrt{-g}\left(\frac{f(R, \mathcal{G})}{2 \kappa^{2}}+\mathcal{L}_{(m)}\right),
$$

where $R$ is the Ricci scalar, $\kappa=\frac{1}{M_{\mathrm{P}}}$ is the gravitational constant (where $M_{\mathrm{P}}$ denotes the reduced Planck mass), and
$\mathcal{G}$ signifies the Gauss-Bonnet invariant defined as $\mathcal{G}=R^{2}-4 R_{\alpha \beta} R^{\alpha \beta}+R_{\alpha \beta \gamma \delta} R^{\alpha \beta \gamma \delta}$, with $R_{\alpha \beta}$ and $R_{\alpha \beta \gamma \delta}$ being the Ricci and Riemann tensor, respectively. Last, $\mathcal{L}_{(m)}$ is the Lagrangian density of the perfect matter fluids, which contains all of the information for non-relativistic matter (CDM), and relativistic matter (radiation). Furthermore, we assume that the cosmological background corresponds to that of a flat Friedman-Robertson-Walker (FRW) metric, with the line element being

$$
d s^{2}=-d t^{2}+a^{2}(t) \delta_{i j} d x^{i} d x^{j},
$$

where $a(t)$ denotes the scale factor. As a result, the Ricci and Gauss-Bonnet scalars are reduced to simpler forms, which read

$$
\begin{gathered}
R=12 H^{2}+6 \dot{H}, \\
\mathcal{G}=24 H^{2}\left(\dot{H}+H^{2}\right),
\end{gathered}
$$

where $H$ signifies the Hubble parameter defined as $H=\frac{\dot{a}}{a}$ and, as usual, a dot denotes differentiation with respect to cosmic time $t$. Thus, by varying the gravitational action (1) with respect to the metric tensor $g^{\mu \nu}$, we can derive the gravitational field equation. Here we separate our equations into space and time components, and hence the equations of motion are

$3 f_{R} H^{2}=\kappa^{2} \rho_{(m)}+\frac{f_{R} R+f_{\mathcal{G}} \mathcal{G}-f-6 H \dot{f}_{R}-24 H^{3} \dot{f}_{\mathcal{G}}}{2}$,

$$
\begin{aligned}
-2 f_{R} \dot{H}= & \kappa^{2}\left(\rho_{(m)}+P_{(m)}\right)+\ddot{f}_{R}-H \dot{f}_{R}-4 H^{3} \dot{f}_{\mathcal{G}} \\
& +8 H \dot{H} \dot{f}_{\mathcal{G}}+4 H^{2} \ddot{f}_{\mathcal{G}},
\end{aligned}
$$

where for simplicity we denote differentiation with respect to a scalar function with a subscript. Furthermore, as stated before, the matter density is comprised of both relativistic and nonrelativistic particles and consequently is written as

$$
\rho_{(m)}=\rho_{d 0}\left(\frac{1}{a^{3}(t)}+\chi \frac{1}{a^{4}(t)}\right),
$$

where $\rho_{d 0}$ signifies the current value of the nonrelativistic density and $\chi=\frac{\rho_{r 0}}{\rho_{d 0}}$ is the ratio of the current values of relativistic and nonrelativistic matter. In addition, $P_{(m)}$ denotes the corresponding pressure, which is connected to the matter density as

$$
\begin{aligned}
P_{i} & =\omega_{i} \rho_{i}, \\
P_{(m)} & =\sum_{i} P_{i},
\end{aligned}
$$


with $\omega_{i}$ being the equation-of-state parameter for a specific kind of matter and $i=d, r$, with the subscript " $i$ " indicating either non-relativistic (d) or relativistic matter (r) perfect fluids. Both kinds of fluids are treated as barotropic perfect fluids with continuity equations

$$
\dot{\rho}_{i}+3 H\left(\rho_{i}+P_{i}\right)=0 .
$$

The aim of our study is to derive a functional form for the Hubble parameter, and hence only a single equation of motion is necessary. In the following, we aim to solve Eq. (5) numerically and use it to extract the form of the Hubble rate during the dark energy era. Before we continue, however, we make certain changes which will facilitate our study. Specifically, we use the redshift as a dynamical variable, and we also introduce a statefinder variable $y_{H}(z)$ (which we will define shortly) in order to make the late-time study more concrete and easy to tackle numerically.

So the cosmic time will be replaced by a more convenient variable, which is the redshift $z$. From the definition of the redshift,

$$
1+z=\frac{1}{a(t)},
$$

where we assume that at the present time the scale factor is equal to unity, a new differential operator can be constructed by simply performing a differentiation on this particular relation, which in turn reads

$$
\frac{d}{d t}=-H(1+z) \frac{d}{d z},
$$

where now the Hubble parameter depends solely on the redshift, that is, $H=H(z)$. This operator is of paramount importance as each object in the equations of motion that is differentiated with respect to the cosmic time can be transformed into a redshift-dependent quantity by using the above transformation. Below we quote some important

quantities that will be used frequently in this paper, and these are transformed as

$$
\begin{gathered}
\dot{H}=-H(1+z) H^{\prime} \\
\dot{R}=6 H(1+z)^{2}\left(H^{\prime 2}+H H^{\prime \prime}-\frac{3 H H^{\prime}}{1+z}\right), \\
\dot{\mathcal{G}}=24(1+z)^{2} H^{3}\left(3 H^{\prime 2}+H H^{\prime \prime}-\frac{3 H H^{\prime}}{1+z}\right),
\end{gathered}
$$

where a prime denotes differentiation with respect to the redshift. Also, we have

$$
\dot{f}_{X}=-H(1+z) f_{X}^{\prime}, \quad \dot{f}_{X}=\sum_{Y} \dot{Y} f_{X Y},
$$

where $X, Y$ take the values $R, \mathcal{G}$. This is because

$$
\frac{d}{d t}=\frac{d R}{d t} \frac{\partial}{\partial R}+\frac{d \mathcal{G}}{d t} \frac{\partial}{\partial \mathcal{G}},
$$

since $R=R(t), \quad \mathcal{G}=\mathcal{G}(t), \quad$ and $\quad f=f(R, \mathcal{G})$. Both approaches are valid so the choice is up to the reader; however, even for the first case, a similar relation for the differential operator with respect to redshift applies.

The second change that we make is a function replacement; specifically, instead of using the Hubble rate, we use an appropriate statefinder function related to it. But before we continue, it is worth making certain changes in the equations of motion. Recalling Eq. (5), we treat each geometric term derived from the expression $f(\mathcal{G})$ in the gravitational action (1) as a fluid, corresponding to dark energy, which will turn out to be a perfect fluid as well. Assuming that

$$
\rho_{\mathrm{DE}}=\frac{f_{R} R+f_{\mathcal{G}} \mathcal{G}-f-6 H \dot{f}_{R}-24 H^{3} \dot{f}_{\mathcal{G}}}{2 \kappa^{2}}+\frac{3 H^{2}}{\kappa^{2}}\left(1-f_{R}\right),
$$

$$
P_{\mathrm{DE}}=\frac{\ddot{f}_{R}+2 H \dot{f}_{R}-3 H^{2}\left(1-f_{R}\right)+8 H^{3} \dot{f}_{\mathcal{G}}+8 H \dot{H} \dot{f}_{\mathcal{G}}+4 H^{2} \ddot{f}_{\mathcal{G}}}{\kappa^{2}}+\frac{f-f_{R} R-f_{\mathcal{G}} \mathcal{G}}{2 \kappa^{2}}+\frac{2 \dot{H}}{\kappa^{2}}\left(f_{R}-1\right),
$$

the continuity equation reads

$$
\dot{\rho}_{\mathrm{DE}}+3 H\left(\rho_{\mathrm{DE}}+P_{\mathrm{DE}}\right)=0
$$

As mentioned before, this continuity equation implies that the dark energy fluid is perfect, as is the case with the rest of the perfect fluids present. Consequently, the equations of motion obtain the familiar form of the Friedmann and Raychaudhuri equations,

$$
H^{2}=\frac{\kappa^{2}}{3}\left(\rho_{(m)}+\rho_{\mathrm{DE}}\right)
$$

$$
\dot{H}=-\frac{\kappa^{2}}{2}\left(\rho_{(m)}+P_{(m)}+\rho_{\mathrm{DE}}+P_{\mathrm{DE}}\right) .
$$

With these equations at hand, we define the new statefinder function $y_{H}(z)$ as 


$$
y_{H}(z)=\frac{\rho_{\mathrm{DE}}}{\rho_{d 0}} .
$$

This is the new dimensionless function that will participate in the equations of motion instead of the Hubble parameter. Since the dark energy density was defined in Eq. (18), this particular function is related to the Hubble rate as follows:

$$
y_{H}(z)=\frac{H^{2}}{m_{s}^{2}}-\frac{\rho_{(m)}}{\rho_{d 0}},
$$

where $m_{s}$ is a mass scale defined as $m_{s}^{2}=\frac{\kappa^{2} \rho_{d 0}}{3}=H_{0}^{2} \Omega_{(m)}^{0}$, where $H_{0}$ is the current value of the Hubble parameter and $\Omega_{(m)}^{0}$ is the current value of the matter density parameter. Their values will be assumed to be equal to $H_{0}=67.4 \pm$ $0.5 \frac{\mathrm{km}}{\sec \times \mathrm{Mpc}}$ and $\Omega_{(m)}^{0}=0.3153$, which are both based on the latest Planck 2018 data [66]. This statefinder function will be used as a replacement for the Hubble parameter and its derivatives, as shown below:

$$
\begin{gathered}
H^{2}=m_{s}^{2}\left(y_{H}+\frac{\rho_{(m)}}{\rho_{d 0}}\right), \\
H H^{\prime}=\frac{m_{s}^{2}}{2}\left(y_{H}^{\prime}+\frac{\rho_{(m)}^{\prime}}{\rho_{d 0}}\right), \\
H^{\prime 2}+H H^{\prime \prime}=\frac{m_{s}^{2}}{2}\left(y_{H}^{\prime \prime}+\frac{\rho_{(m)^{\prime \prime}}}{\rho_{d 0}}\right) .
\end{gathered}
$$

An observant reader might notice that the Hubble parameter derivatives participate in the previous equations with these exact forms. Indeed, the Ricci scalar and GaussBonnet invariant time derivative contain the above expressions, so with this designation all of the previous equations can be easily rewritten. Furthermore, recalling Eqs. (7) and (11), the density of matter is rewritten with respect to the redshift variable as follows:

$$
\rho_{(m)}=\rho_{d 0}\left((1+z)^{3}+\chi(1+z)^{4}\right),
$$

where $\chi \simeq 3.1 \times 10^{-4}$, and $\chi$ is the fraction of the present day energy density of the radiation over the cold dark matter fluids. Finally, we define the following parameters, which we evaluate during the late-time era. The dark energy equation-of-state $(\mathrm{EoS})$ parameter is equal to

$$
\omega_{\mathrm{DE}}=-1+\frac{1+z}{3} \frac{d \ln y_{H}}{d z},
$$

while the dark energy density parameter is

$$
\Omega_{\mathrm{DE}}=\frac{y_{H}}{y_{H}+\frac{\rho_{(m)}}{\rho_{d 0}}}=y_{H}\left(\frac{m_{s}}{H}\right)^{2} .
$$

Thus, the aim of this paper is to study some appropriate $f(R, \mathcal{G})$ models and compare their behavior with the
$\Lambda \mathrm{CDM}$ model. In order to extract the late-time behavior of each $f(R, \mathcal{G})$ model chosen, we numerically solve the differential equation (5) with respect to the statefinder $y_{H}(z)$ for appropriately chosen, physically motivated initial conditions.

\section{III. $\boldsymbol{R}+\boldsymbol{f}(\mathcal{G})$ GRAVITY: A SINGULAR BOUNCE AT EARLY TIMES AND A DARK ENERGY ERA AT LATE TIMES}

For an arbitrary $f(R, \mathcal{G})$, the possibility of ghosts being present is nonzero. We assume that the first model is ghost free, where the function $f(R, \mathcal{G})$ is replaced by $R+f(\mathcal{G})$. As a result, Eq. (5) is rewritten as

$$
\frac{3 H^{2}}{\kappa^{2}}=\rho_{(m)}+\frac{\mathcal{G} f_{\mathcal{G}}-f-24 \dot{f}_{\mathcal{G}} H^{3}}{2 \kappa^{2}} .
$$

This is the general differential equation that must be solved in the redshift interval $[-0.9,10]$ with respect to $y_{H}(z)$ defined in Eq. (24). Now let us assume that the GaussBonnet function is given by the following expression:

$$
f(\mathcal{G})=\frac{c_{1}}{\mathcal{G}}+c_{2} \mathcal{G}^{\frac{\alpha}{3 \alpha-1}},
$$

where $c_{1}$ and $c_{2}$ are auxiliary constants with mass dimensions $[m]^{6}$ and $[m]^{2-\frac{4 \alpha}{3 \alpha-1}}$, respectively (for consistency), while $\alpha$ is an additional parameter that we assume satisfies the condition $\alpha>1$. This is an interesting model due to the fact that for small values of $\mathcal{G}$, the inverse term $\frac{c_{1}}{\mathcal{G}}$ becomes dominant during late times, whereas $c_{2} \mathcal{G}^{\frac{\alpha}{3 \alpha-1}}$ is dominant during early times when the exponent $\frac{\alpha}{3 \alpha-1}$ is less than unity given that the condition $\alpha>1$ holds true. Therefore, this model is capable of describing both the early- and late-time eras and thus unifying them smoothly.

The singular bounce cosmology is basically realized by an $R+f(\mathcal{G})$ gravity of the form $f(\mathcal{G}) \sim \mathcal{G}^{\frac{1-2 \alpha}{3 \alpha-1}}$, as it was shown in Ref. [52]. This particular $f(\mathcal{G})$ gravity was able to realize a type IV singular bounce with a Hubble rate $H(t) \sim\left(t-t_{s}\right)^{\alpha}$, where $\alpha$ is strictly greater than unity (that is, $\alpha>1)$ and $t_{s}$ is the cosmic time instant at which the singular bounce occurs. From Eq. (32) it is apparent that for $\alpha>1$ the term $\sim \mathcal{G}^{\frac{\alpha}{3 \alpha-1}}$ is dominant at early times, and the term $\sim G^{-1}$ is subdominant during the early-time era. We shall quantify this shortly, but let us briefly discuss the singular bounce generated by the term $\sim \mathcal{G}^{\frac{\alpha}{3 \alpha-1}}$. Following Ref. [52], the primordial curvature perturbations are generated near the bouncing point $t=t_{s}$, and exit the Hubble horizon after the singular bouncing point. The Hubble rate near the bouncing point could be of the order of $H_{I} \sim$ $10^{13} \mathrm{GeV}$ (borrowing the value of the Hubble rate from low-scale inflation studies), and thus the term $\sim \mathcal{G}^{\frac{\alpha}{3 \alpha-1}}$ for $\alpha=3.30579$ and $c_{2}=1 \mathrm{eV}^{2-\frac{4 \alpha}{3 \alpha-1}}$ (which are the values of the parameters $\alpha$ and $c_{2}$ we use in the following) is of the 
order of $c_{2} \mathcal{G}^{\frac{\alpha}{3 \alpha-1}} \sim 1.11887 \times 10^{30} \mathrm{eV}^{2}$, while the term $c_{1} \mathcal{G}^{-1}$ is of the order of $c_{1} \mathcal{G}^{-1} \sim 10^{-88} \mathrm{eV}^{2}$, for $c_{1}=1 \mathrm{eV}^{6}$. Thus, the term $\sim G^{-1}$ is indeed significantly subdominant during the early-time era, near the singular bouncing point. In Ref. [52] we calculated in detail the power spectrum of the primordial scalar curvature perturbations, and we found that it is equal to

$$
\mathcal{P}_{R} \sim k^{\frac{7}{2}+3+\frac{\left(2-2 \alpha+\alpha^{2}\right) \mu}{2(-1+\alpha)}} .
$$

From the above expression we easily derived the spectral index of the primordial curvature perturbations, which is equal to

$n_{s}-1 \equiv \frac{d \ln \mathcal{P}_{\mathcal{R}}}{d \ln k}=\frac{7}{2}+3+\frac{2-2 \alpha+\alpha^{2}}{2(\alpha-1)} \mu=1-\frac{11}{2(\alpha-1)^{2}}$.

It is easy to see that for $\alpha$ taken in the range $\alpha=[3.2999,3.31]$, the spectral index becomes compatible with the latest Planck 2018 data [67], which constrain the spectral index to be $n_{s}=0.9649 \pm 0.0042$. Also, for $\alpha<-1$ the spectral index can be compatible with the Planck data; however, the $\alpha<-1$ case corresponds to a big rip singularity, and thus it is not physically acceptable. The singular bounce with a type IV singularity is more physically appealing, since the type IV singularity is quite smooth and does not affect any physical quantities that can be defined on the three-dimensional spacelike hypersurface which is defined at the instant that the singularity occurs.

Having determined that the term practically generates the singular bounce at early times and is dominant during this primordial era, let us see how things are modified at late times. Apparently, the late-time era is controlled by the term $\sim \mathcal{G}^{-1}$, but let us see this explicitly in a quantitative way for the moment. Let us use the current value of the Hubble rate, which is $H_{0} \sim 10^{-33} \mathrm{eV}$, so the term $\mathcal{G}^{\frac{\alpha}{3 \alpha-1}}$ is approximately of the order of $c_{2} \mathcal{G}^{\frac{\alpha}{3 \alpha-1}} \sim 8.44947 \times 10^{-46} \mathrm{eV}^{2}$, while the term $c_{1} \mathcal{G}^{-1} \sim 10^{132} \mathrm{eV}^{2}$. Thus, it is quantitatively apparent that the term $\sim \mathcal{G}^{-1}$ is quite dominant at late times and controls the evolution.

In this section we numerically demonstrate that the term $\mathcal{G}^{-1}$ indeed dominates the late-time era, generating a viable dark energy era. Our results are robust to changes of the parameter $\alpha$ for all values larger than unity, but the analysis will focus on those values of the parameter $\alpha$ that yield a spectral index of the primordial curvature perturbations compatible with the Planck 2018 data, i.e., $\alpha=3.30579$. So let us proceed with the analysis of the model and express all of the differential equations and physical quantities in terms of the statefinder quantity $y_{H}(z)$. Before going into the details of our analysis, let us quote some useful expressions. For the $f(\mathcal{G})$ gravity chosen as in Eq. (32), we have

$$
\begin{gathered}
f_{\mathcal{G}}=-\frac{c_{1}}{\mathcal{G}^{2}}+\frac{c_{2} \alpha}{3 \alpha-1} \mathcal{G}^{\frac{1-2 \alpha}{\beta \alpha-1}} \\
\dot{f}_{\mathcal{G}}=\dot{\mathcal{G}}\left(\frac{2 c_{1}}{\mathcal{G}^{3}}+\frac{c_{2} \alpha(1-2 \alpha)}{(3 \alpha-1)^{2}} \mathcal{G}^{\frac{2-5 \alpha}{3 \alpha-1}}\right),
\end{gathered}
$$

and thus Eq. (5) reads

$$
\begin{aligned}
3 H^{2}= & \kappa^{2} \rho_{(m)}+\frac{2 c_{1}(1-3 \alpha)+c_{2}(2 \alpha-1) \mathcal{G}^{\frac{4 \alpha-1}{3 \alpha-1}}}{2 \mathcal{G}(3 \alpha-1)} \\
& -12 \dot{\mathcal{G}}\left(\frac{2 c_{1}}{\mathcal{G}^{3}}+\frac{c_{2} \alpha(1-2 \alpha) \mathcal{G}^{\frac{2-5 \alpha}{3 \alpha-1}}}{(3 \alpha-1)^{2}}\right) H^{3} .
\end{aligned}
$$

This particular differential equation will be solved numerically ${ }^{1}$ for redshifts in the range $z=[-0.9,10]$, but for the statefinder function $y_{H}(z)$ introduced as a replacement for the Hubble parameter in Eq. (24). The initial conditions we choose for the statefinder $y_{H}(z)$ are $y_{H}(z=10)=\frac{\Lambda}{3 m_{s}^{2}}(1+$ $\left.\frac{1+z_{f}}{100}\right)$ and $y_{H}^{\prime}(z=10)=\frac{\Lambda}{3 m_{s}^{2}} \frac{1}{1000}$ for $z_{f}=10$, and there is a strong physical motivation for using these initial conditions; see, for example, Ref. [42]. In addition, the choice for $\Lambda$ is $\Lambda=1.1895 \times 10^{-66} \mathrm{eV}$ while the mass scale $m_{s}$ is $m_{s}=4.32552 \times 10^{-34} \mathrm{eV}$, and in addition in the following we take $c_{1}=1 \mathrm{eV}^{6}, c_{2}=1 \mathrm{eV}^{2-\frac{4 \alpha}{3 \alpha-1}}$, and $\alpha=3.30579$. Then, by numerically solving the differential equation (37) using the aforementioned initial conditions and values for the free parameters, we can analyze several statefinder quantities of cosmological interest for the late-time era, and we will compare the results with the $\Lambda \mathrm{CDM}$ model and an $f(R)$ gravity model that is known to produce viable solutions for the late-time era. The comparison of the results of the Gauss-Bonnet model with the $\Lambda \mathrm{CDM}$ is obvious, since the latter is the cornerstone model of latetime phenomenology, since it is highly compatible with the cosmic microwave background (CMB). However, we need to discuss the comparison of the Gauss-Bonnet model with the $f(R)$ gravity theory, since these are apparently two distinct and phenomenologically competing theories. Our motivation is simply to investigate whether the dark energy oscillations at large redshifts $(z \geq 4)$ persist in the GaussBonnet theory case. Our results are quite interesting, since in the Gauss-Bonnet case the oscillations do not occur. Also, we calculate the predicted values for some quantities of cosmological interest and compare these values with the latest constraints from the Planck Collaboration on these cosmological parameters [66]. For our analysis, we use the CMB-based value for the Hubble rate, which is [66]

$$
H_{0}=67.4 \pm 0.5 \frac{\mathrm{km}}{\mathrm{sec} \times \mathrm{Mpc}},
$$

\footnotetext{
${ }^{1}$ We used Mathematica $11.3{ }^{\circledR}$
} 
so $H_{0}=67.4 \mathrm{~km} / \mathrm{sec} / \mathrm{Mpc}$, which is $H_{0}=1.37187 \times$ $10^{-33} \mathrm{eV}$. Also, let us briefly discuss the cosmological quantities and statefinders that we analyze and compare in this paper, for the $\Lambda \mathrm{CDM}$, Gauss-Bonnet, and $f(R)$ gravity models. An important quantity is the dark energy EoS parameter, defined as $\omega_{\mathrm{DE}}=\frac{P_{\mathrm{DE}}}{\rho_{\mathrm{DE}}}$, which in terms of the statefinder quantity $y_{H}(z)$ is expressed as follows:

$$
\omega_{\mathrm{DE}}(z)=-1+\frac{1}{3}(z+1) \frac{1}{y_{H}(z)} \frac{d y_{H}(z)}{d z} .
$$

Basically, the above quantity is itself a statefinder quantity, since it depends on the geometry through its explicit dependence on the Hubble rate derivatives. Another important cosmological quantity is the dark energy density parameter $\Omega_{\mathrm{DE}}(z)$, defined as $\Omega_{\mathrm{DE}}=\frac{\rho_{\mathrm{DE}}}{\rho_{\mathrm{tot}}}$, which in terms of the statefinder quantity $y_{H}(z)$ is written as

$$
\Omega_{\mathrm{DE}}(z)=\frac{y_{H}(z)}{y_{H}(z)+(z+1)^{3}+\chi(z+1)^{4}} .
$$

Now, for the comparisons with the $\Lambda \mathrm{CDM}$ model, the Hubble rate for the $\Lambda \mathrm{CDM}$ model is equal to

$$
H_{\Lambda}(z)=H_{0} \sqrt{\Omega_{\Lambda}+\Omega_{M}(z+1)^{3}+\Omega_{r}(1+z)^{4}},
$$

where again $H_{0}$ is the value of the Hubble rate at present time, namely, $H_{0} \simeq 1.37187 \times 10^{-33} \mathrm{eV}$ [66], while $\Omega_{\Lambda} \simeq 0.681369$, and finally $\Omega_{M} \sim 0.3153$ [66]. Also, $\Omega_{r} / \Omega_{M} \simeq \chi$, where we defined the parameter $\chi$ below Eq. (28).

Finally, we examine four statefinder quantities in this late-time study: the deceleration parameter $q$, the jerk $j$, the snap parameter $s$, and finally the parameter $\operatorname{Om}(z)$, which in terms of the Hubble rate are defined as

$$
\begin{gathered}
q=-1-\frac{\dot{H}}{H^{2}}, \\
j=\frac{\ddot{H}}{H^{3}}-3 q-2, \\
s=\frac{j-1}{3\left(q-\frac{1}{2}\right)}, \\
O m(z)=\frac{\left(\frac{H(z)}{H_{0}}\right)^{2}-1}{(1+z)^{3}-1} .
\end{gathered}
$$

Of course, the first three must be expressed in terms of the redshift variable, so for simplicity we quote the simplest expressions of the three:

$$
q=-1+(1+z) \frac{H^{\prime}}{H},
$$

$$
j=(1+z)^{2}\left(\left(\frac{H^{\prime}}{H}\right)^{2}+\frac{H^{\prime \prime}}{H}-\frac{2 H^{\prime}}{(1+z) H}\right)+1 .
$$

For the $\Lambda \mathrm{CDM}$ model, the statefinders $j, s$, and $\mathrm{Om}(z)$ have the following simple values: $s=0, j=1$, and $\operatorname{Om}(z)=\Omega_{M} \simeq 0.3153$. Let us now proceed to the results of our numerical analysis. First, we compare the GaussBonnet model with the $\Lambda$ CDM model, and in Fig. 1 we present the comparisons of the deceleration parameter (upper left), the jerk (upper right), the snap (bottom left), and the parameter $\operatorname{Om}(z)$ (bottom right) for the GaussBonnet model (blue curves) and $\Lambda \mathrm{CDM}$ model (red curves). As is obvious, in the case of the deceleration parameter the two curves are indistinguishable, while differences can be found for the other three statefinders. Also, it is worth mentioning that for the snap parameter, for redshifts $z \sim 4$ and smaller, the $\Lambda \mathrm{CDM}$ and Gauss-Bonnet models are indistinguishable.

Now in order to investigate the dark energy oscillations known to affect $f(R)$ gravity theories, we present the results of our numerical analysis for the Gauss-Bonnet theory, focusing on the dark energy $\operatorname{EoS} \omega_{\mathrm{DE}}$ and the dark energy density parameter $\Omega_{\mathrm{DE}}(z)$, the behaviors of which are plotted in Fig. 2 for the pure Gauss-Bonnet theory. As can be seen in Fig. 2, no dark energy oscillations occur, but in order to make this result more clear we compare the Gauss-Bonnet theory with a viable $f(R)$ gravity theory, the functional form of which is [42]

$$
f(R)=R+\left(\frac{R}{M}\right)^{2}-\gamma \Lambda\left(\frac{R}{3 m_{s}^{2}}\right)^{\delta},
$$

where, $M$ is an auxiliary parameter with mass dimension $[\mathrm{m}]$ and is given by the expression $M=1.5 \times 10^{-5} \frac{50}{N} M_{P}$, where $N$ is the number of $e$-foldings corresponding to the inflationary era $(N=60)$ and $M_{\mathrm{P}}$ is the reduced Planck mass. Essentially, the $R^{2}$ term contributes to the inflationary era and early times, whereas $\left(\frac{R}{3 m_{s}^{2}}\right)^{\delta}$ for $\delta<1$ becomes dominant in the late-time era. In Fig. 3 we plot the behaviors of the dark energy EoS $\omega_{\mathrm{DE}}$ (left) and the dark energy parameter $\Omega_{\mathrm{DE}}$ (right) for the $f(R)$ model (red curves) and Gauss-Bonnet gravity (blue curves). As is apparent, the behavior of the dark energy density parameter is indistinguishable between the models; however, the dark energy EoS for the $f(R)$ gravity model has strong oscillations for $z \geq 4$, which are completely absent in the Gauss-Bonnet model. Thus, the dark energy oscillation issue that haunts $f(R)$ gravity models is absent in the Gauss-Bonnet models. This claim is further supported by the plots appearing in Figs. 4 and 5. In Fig. 4 we compare the deceleration parameter $q$ and the statefinder $y_{H}(z)$ for the $f(R)$ gravity model (red curves) and the Gauss-Bonnet model (blue). The absence of oscillations in both cases is obvious for the Gauss-Bonnet case, and the same conclusions can be derived if we look at Fig. 5 where we plot the 

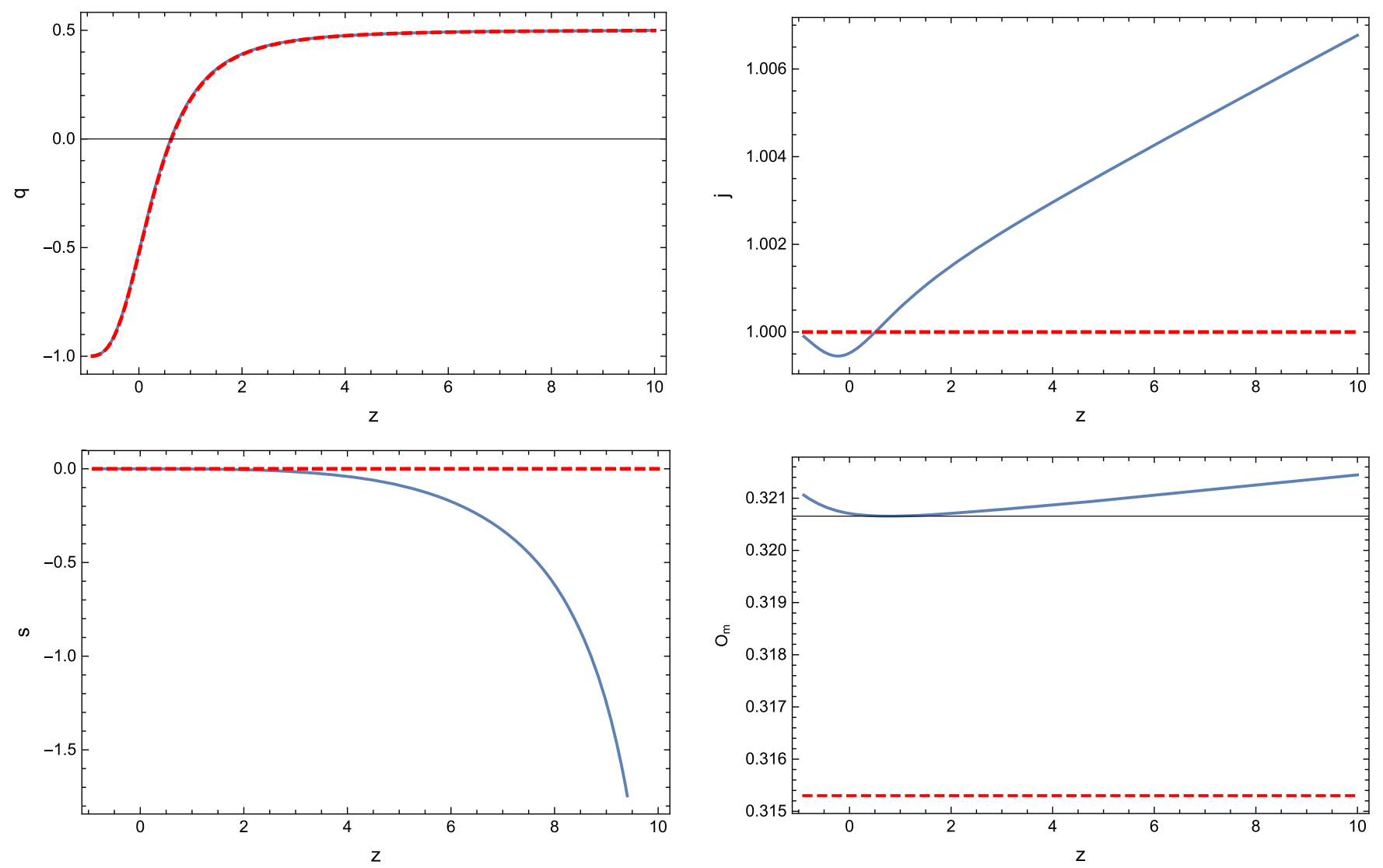

FIG. 1. Comparison of several statefinder quantities for $R+f(\mathcal{G})$ gravity and the $\Lambda$ CDM model.

statefinder parameters $j(z)$ (upper left), $s(z)$ (upper right), and $O m(z)$ (bottom) for the $f(R)$ gravity model (red curves) and Gauss-Bonnet gravity (blue curves). In the upper diagrams, it becomes apparent that the main difference lies in the dark energy oscillations which, as expected, are enhanced in higher-order derivatives of $y_{H}$ for the $f(R)$ gravity case.

Finally, in order to have a concrete idea of how well the Gauss-Bonnet model behaves, we compare the values of several statefinders for the $\Lambda \mathrm{CDM}$ model and GaussBonnet gravity, and also directly compare the dark energy EoS and dark energy density parameters at present time for the Gauss-Bonnet model with the latest Planck data. Our results are summarized in Table I. As can be seen in Table I, the statefinder values for the Gauss-Bonnet model are quite close to the corresponding $\Lambda \mathrm{CDM}$ values, and in addition the Gauss-Bonnet model value for the dark energy density
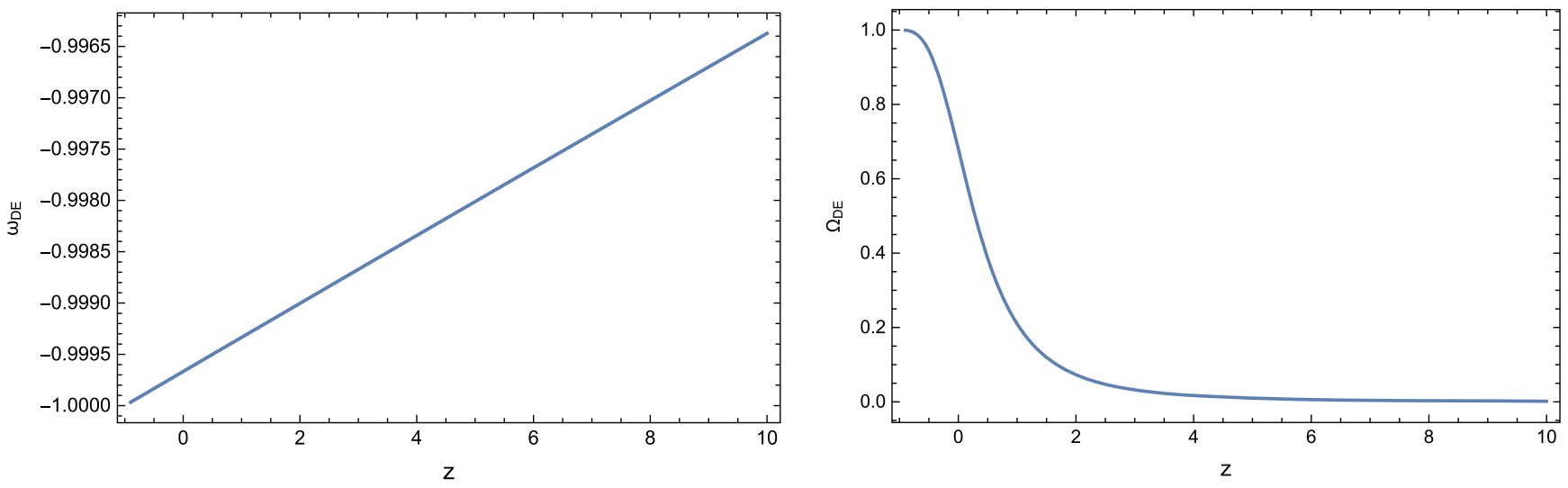

FIG. 2. Dark energy EoS parameter $\omega_{\mathrm{DE}}$ (left) and dark energy density parameter $\Omega_{\mathrm{DE}}$ (right) for the $R+f(\mathcal{G})$ model. In this case, it can be easily seen that the EoS of the dark energy density parameter is slowly varying near $\omega_{\mathrm{DE}}=-1$, while the density parameter $\Omega_{\mathrm{DE}}$ increases until it reaches the value $\Omega_{\mathrm{DE}}=1$. 

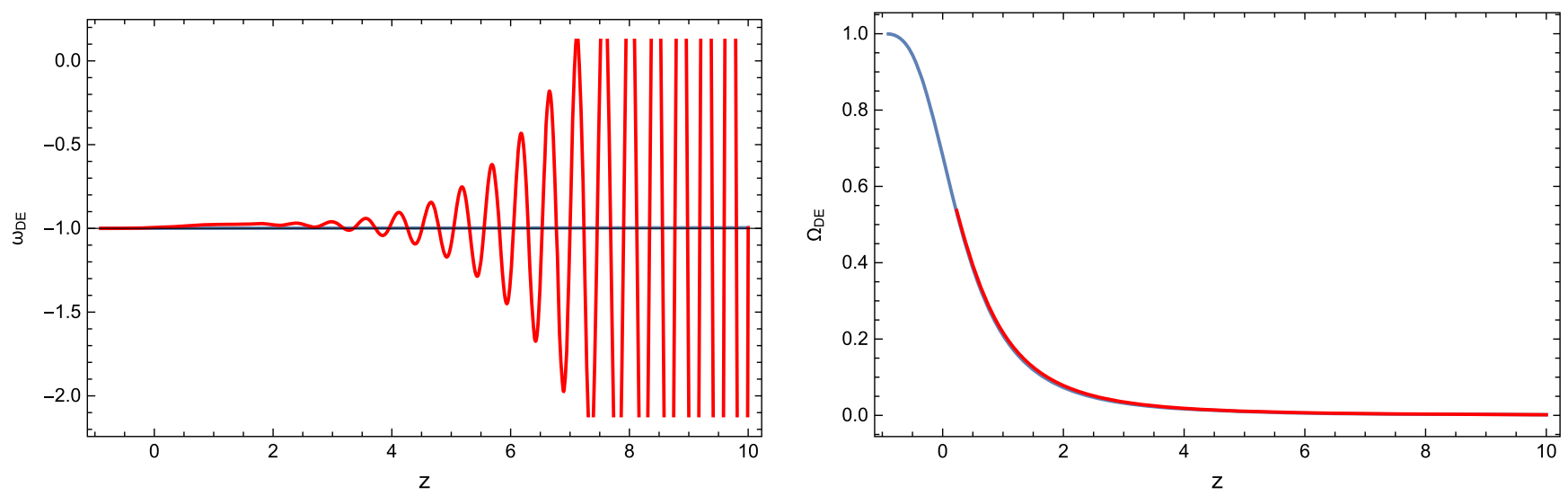

FIG. 3. Dark energy EoS parameter $\omega_{\mathrm{DE}}$ (left) and the dark energy density parameter $\Omega_{\mathrm{DE}}$ (right) for the $f(R)$ and $R+f(\mathcal{G})$ cases, depicted with red and blue curves, respectively.

parameter is $\Omega_{\mathrm{DE}}(0)=0.679553$, which is compatible with the latest Planck constraint $\Omega_{\mathrm{DE}}=0.6847 \pm 0.0073$ [66]. In addition, the dark energy EoS parameter value for the Gauss-Bonnet model at present time is $\omega_{\mathrm{DE}}(0)=$ -0.999667 , which is in good agreement with the corresponding Planck constraint $\omega_{\mathrm{DE}}=-1.018 \pm 0.031$ [66]. In conclusion, the Gauss-Bonnet model of Eq. (32) is able to produce a phenomenologically viable early-time bounce, while at late times it produces a viable dark energy era, which is in addition free from dark energy oscillations. In the next section we also discuss a more complicated $f(R, \mathcal{G})$ model that is, however, less appealing in comparison to the model we presented in this section, due to the primordial ghost modes in those $f(R, \mathcal{G})$ models.

\section{A. On the stability of the $F(G)$ gravity solutions and cosmological perturbations in the vicinity of the bouncing point}

Let us now investigate the stability of the $f(\mathcal{G})$ gravity solutions and discuss the stability of the cosmological

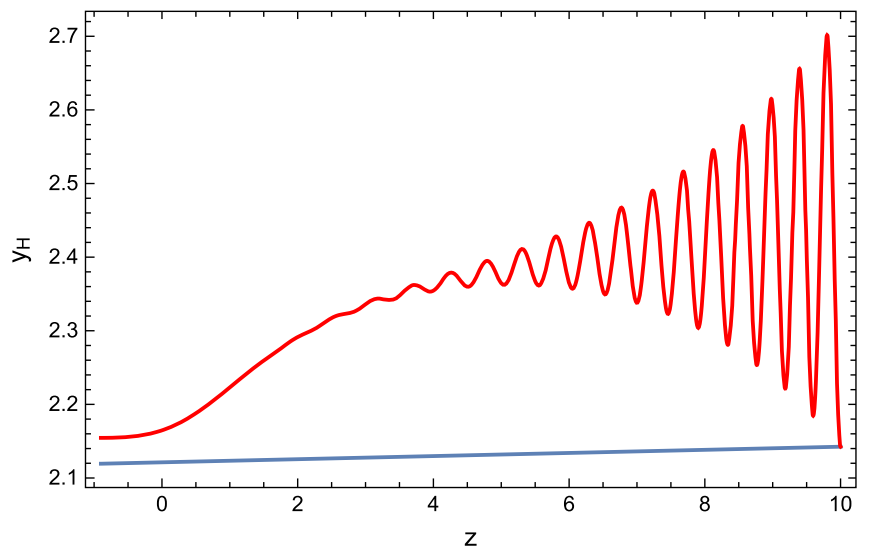

perturbations as the general-relativistic limit is approached. The FRW equations for $f(\mathcal{G})$ gravity constitute a dynamical system, and the stability of the solutions can be examined if we perturb this dynamical system. We consider linear perturbations of the cosmological solutions, as functions of the Hubble rate, and the presence of an instability would indicate that the solution is not the final attractor for the theory at hand. For the era near the bouncing point, this is somewhat expected because the evolution continues after the bouncing point, but for the late-time era the expected behavior is rather vague. This is due to the fact that the latetime era seems to be a de Sitter-like solution by looking at the dark energy EoS parameter, but eventually it is not an exact de Sitter solution. So let us explicitly check the stability of the dynamical system to linear perturbations of the cosmological solutions, in order to shed some light on this issue. The Hubble rate in the vicinity of the bouncing point is

$$
H(t)=\beta\left(t-t_{s}\right)^{\alpha},
$$

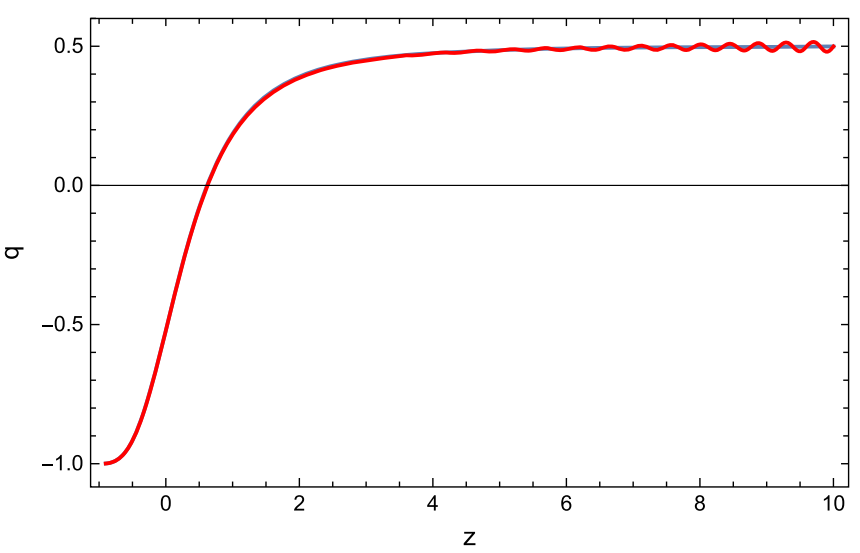

FIG. 4. Comparison between the $f(R)$ (red) and $R+f(\mathcal{G})$ cases (blue) for the parameters $y_{H}(z)$ (left) and $q(z)$ (right). While the statefinder $y_{H}$ is completely different between the two models, the deceleration parameter seems to be the same when dark energy oscillations are not present, i.e., for $z \leq 5$. 

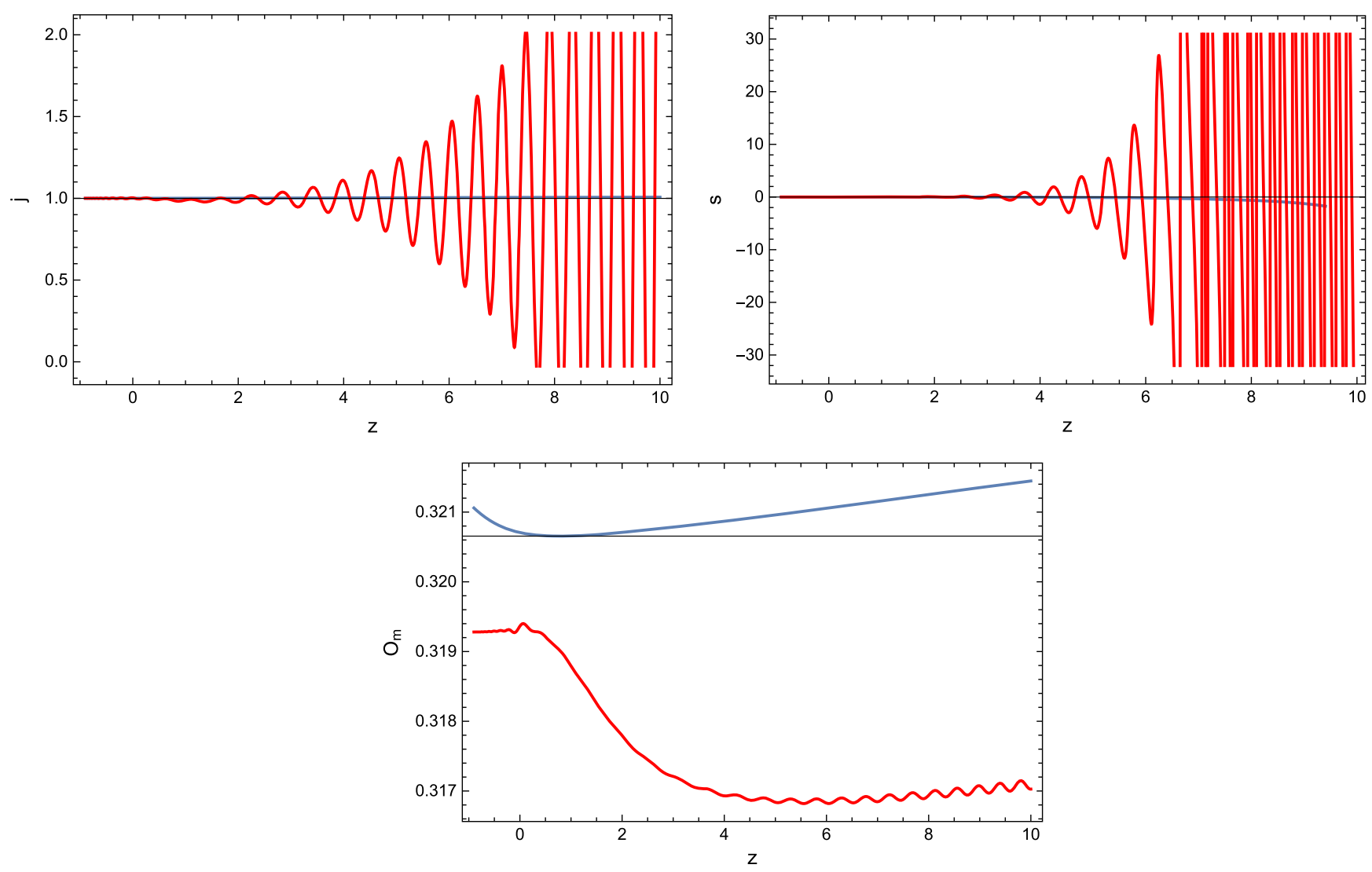

FIG. 5. Statefinder parameters $j(z)$ (upper left), $s(z)$ (upper right), and $O m(z)$ (bottom) for the $f(R)$ (red) and $R+f(\mathcal{G})$ (blue) models. In the upper diagrams, it becomes apparent that the main difference lies in the dark energy oscillations which (as expected) are enhanced in higher-order derivatives of $y_{H}$.

and the $f(\mathcal{G})$ gravity that approximately realizes the Hubble rate (49) is

$$
f(\mathcal{G}) \sim c_{2} \mathcal{G}^{\frac{\alpha}{3 \alpha-1}} .
$$

Following the strategy of Ref. [68], we linearly perturb the solution of the Friedmann equation $g(N)=H^{2}$ in the following way:

$$
g(N) \rightarrow g(N)+\delta g(N)
$$

where $g(N)$ is a solution of the Friedmann equation and $N$ is the number of $e$-foldings. By using the function $g(N)$, the Friedmann equation can be cast in the following form:

$$
\begin{aligned}
& 288 g^{2}(N) f^{\prime \prime}(\mathcal{G})\left[\left(\left(g^{\prime}(N)\right)^{2}+g(N)\right) g^{\prime \prime}(N)+4 g(N) g^{\prime}(N)+4 g(N) g^{\prime}(N)\right] 6 g(N) \\
& \quad+f(\mathcal{G})-12 g(N)\left(g^{\prime}(N)+2 g(N)\right) f^{\prime}(\mathcal{G})=0 .
\end{aligned}
$$

Now, the actual conditions that ensure that the dynamical system of Eq. (52) is stable to linear perturbations are [68]

$$
\frac{J_{2}}{J_{1}}>0, \quad \frac{J_{3}}{J_{1}}>0,
$$

where $J_{1}$ is

$$
J_{1}=288 g(N)^{3} f^{\prime \prime}(\mathcal{G}),
$$


$J_{2}$ is

$$
J_{2}=432 g(N)^{2}\left(\left(2 g(N)+g^{\prime}(N)\right) f^{\prime \prime}(\mathcal{G})+8 g(N)\left(g^{\prime}(N)^{2}+g(N)\left(4 g^{\prime}(N)+g^{\prime \prime}(N)\right)\right) f^{\prime \prime}(\mathcal{G})\right),
$$

and finally $J_{3}$ is

$$
\begin{aligned}
J_{3}= & 6\left(1+24 g(N)\left(-8 g(N)^{2}+3 g^{\prime}(N)^{2}+6 g(N)\left(3 g^{\prime}(N)+g^{\prime \prime}(N)\right)\right) f^{\prime \prime}(\mathcal{G})\right. \\
& \left.+24 g(N)\left(4 g(N)+g^{\prime}(N)\right)\left(g^{\prime}(N)^{2}+g(N)\left(4 g(N)+g^{\prime \prime}(N)\right)\right) f^{\prime \prime}(\mathcal{G})\right) .
\end{aligned}
$$

Now let us explicitly calculate the parameters $J_{1}, J_{2}$, and $J_{3}$ for the era corresponding to the bouncing point, so the $f(\mathcal{G})$ gravity is given in Eq. (50). By expressing the Hubble rate (49) as a function of the number of $e$-foldings $N$, with the latter being $N=\ln a$, the function $g(N)$ becomes

$$
g(N)=\frac{\beta^{2} N^{\zeta}}{\delta}
$$

with $\zeta=\frac{2 \alpha}{1+\alpha}$. Now we proceed with the stability conditions, and by calculating the parameters $J_{1}, J_{2}$, and $J_{3}$ we get

$$
\frac{J_{2}}{J_{1}}=\frac{3\left(\delta^{2} N(2 N+\zeta)+8 N^{2 \zeta} \beta^{4} \zeta(-1+4 N+2 \zeta)\right)}{2 \delta^{2} N^{2}},
$$

and $J_{3} / J_{1}$ is

$$
\begin{aligned}
\frac{J_{3}}{J_{1}}= & -\frac{1}{c_{2} \delta \alpha(-1+2 \alpha)} 3^{1+\frac{\alpha}{1-3 \alpha} 4 \frac{\alpha}{1-3 \alpha}} N^{-2+\zeta}(1-3 \alpha)^{2} \beta^{2}(\zeta)^{2}\left(\frac{N^{-1+2 \zeta} \beta^{4}(\zeta)}{\delta^{2}}\right){ }^{\frac{\alpha}{1-3 \alpha}} \\
& \times\left(1+\frac{2^{\frac{1-\alpha}{-1+3 \alpha}} 3^{\frac{1-2 \alpha}{-1+3 \alpha}} c_{2} \delta N^{-\zeta} \alpha(-1+2 \alpha)\left(\frac{N^{-1+2 \zeta} \beta^{4}(2 N+\zeta)}{\delta^{2}}\right)^{\frac{\alpha}{-1+3 \alpha}}\left(8 N^{2}-18 N \zeta+3(2-3 \zeta) \zeta\right)}{(1-3 \alpha)^{2} \beta^{2}(2 N+\zeta)^{2}}\right. \\
& \left.-\frac{2^{\frac{1-\alpha}{1+3 \alpha}} 3^{\frac{1-2 \alpha}{-1+3 \alpha}} c_{2} \alpha(-1+2 \alpha)\left(\frac{N^{-1+2 \zeta} \beta^{4}(\zeta)}{\delta^{2}}\right)^{-\frac{\alpha}{1+3 \alpha}}(\zeta)(\zeta(-1+2 \zeta))}{N(1-3 \alpha)^{2}(\zeta)^{2}}\right)
\end{aligned}
$$

Focusing on the vicinity of the bouncing point $t \rightarrow t_{s}$, which corresponds to $N \rightarrow 0$, we get

$$
\begin{aligned}
& \frac{J_{2}}{J_{1}}=3+\frac{3 \zeta}{2 N}, \\
& \frac{J_{3}}{J_{1}}=-\mathcal{A} N^{-2+\zeta+\frac{\alpha-2 \alpha \zeta}{-1+3 \alpha},}
\end{aligned}
$$

TABLE I. Cosmological parameters of the $R+f(\mathcal{G})$ choice at present time versus the expected value of the $\Lambda \mathrm{CDM}$ model or the Planck 2018 data (where available). It becomes apparent that this particular choice of initial conditions results in values that are quite close to the expected ones.

\begin{tabular}{lcc}
\hline \hline Parameter & Value & $\Lambda$ CDM Value/ Planck 2018 Data \\
\hline$y_{H}(z=0)$ & 2.1213 & $\ldots$ \\
$y_{H}^{\prime}(z=10)$ & 0.002119 & $\ldots$ \\
$q(z=0)$ & -0.51894 & -0.535 \\
$j(z=0)$ & 0.99952 & 1 \\
$j(z=10)$ & 1.00677 & 1 \\
$s(z=0)$ & -0.00015711 & 0 \\
$O m(z=0)$ & 0.320707 & $0.3153 \pm 0.07$ \\
$\Omega_{\mathrm{DE}}(z=0)$ & 0.679553 & $0.6847 \pm 0.0073$ \\
$\omega_{\mathrm{DE}}(z=0)$ & -0.999667 & $-1.018 \pm 0.031$ \\
\hline \hline
\end{tabular}

where the parameter $\mathcal{A}$ is

$$
\mathcal{A}=\frac{3^{1+\frac{\alpha}{1-3 \alpha}} 4 \frac{\alpha}{1-3 \alpha} \delta(1-3 \alpha)^{2} \gamma\left(\frac{\beta^{4} \gamma}{\delta^{2}}\right)^{1+\frac{\alpha}{1-3 \alpha}}}{c_{2} \alpha(-1+2 \alpha) \beta^{2}},
$$

and $\mathcal{A}$ is obviously positive. Hence, $J_{2} / J_{1}>0$ and $J_{3} / J_{1}<0$, and therefore the dynamical system of cosmological equations is unstable near the bouncing point, as we anticipated.

Now let us consider the stability of the cosmological solution for the late-time era, focusing on redshifts $z \sim 0$. Since the late-time evolution results in an EoS for the dark energy that is approximately equal to -1 , the late-time evolution for redshifts $z \sim 0$ can be approximated by a de Sitter evolution, and it is realized by the approximate $f(\mathcal{G})$ gravity of the form

$$
f(\mathcal{G}) \sim c_{1} / \mathcal{G}
$$

Hence, assuming that the late-time Hubble rate has the de Sitter form $H(t) \sim H_{0}$, in effect the function $g(N)$ in this case has the form $g(N)=H_{0}^{2}$. Let us repeat the procedure we followed for the case of the bounce near the bouncing 
point by perturbing the de Sitter vacuum solution $g(N)=$ $H_{0}^{2}$ using a linear perturbation of the form (51); the variable $J_{1}$ becomes

$$
J_{1}=\frac{c_{1}}{24 H_{0}^{6}}
$$

while the parameter $J_{2}$ reads

$$
J_{2}=\frac{c_{1}}{8 H_{0}^{6}},
$$

and finally the parameter $J_{3}$ reads

$$
J_{3}=6\left(-\frac{c_{1}}{36 H_{0}^{6}}+\frac{c_{1}}{18 H_{0}^{4}}+1\right) .
$$

Now the fraction $J_{2} / J_{1}$ is equal to $J_{2} / J_{1}=3$, while the fraction $J_{3} / J_{1}$ is equal to

$$
\frac{J_{3}}{J_{1}}=\frac{144 H_{0}^{6}}{c_{1}}+8 H_{0}^{2}-4,
$$

and since for the late-time analysis we took $H_{0} \simeq$ $1.37187 \times 10^{-33} \mathrm{eV}$ and $c_{1}=1 \mathrm{eV}^{6}$, this means that $J_{3} / J_{1}$ is obviously negative. This means that the dynamical system corresponding to the linear cosmological perturbations is unstable for the late-time solutions. This means that the late-time dynamical system has an unstable de Sitter attractor (fixed point), and thus in the era that lies beyond $z=0$ the system will not remain in the de Sitter vacuum. Physically, this could mean that the late-time dark energy EoS will not remain at the fixed value of -1 , but might evolve to the phantom or quintessence regime. In any case, this result is somewhat interesting since it deviates from the $\Lambda \mathrm{CDM}$ description, in which case the de Sitter state is stable and unchanged, and the cosmological constant is constant.

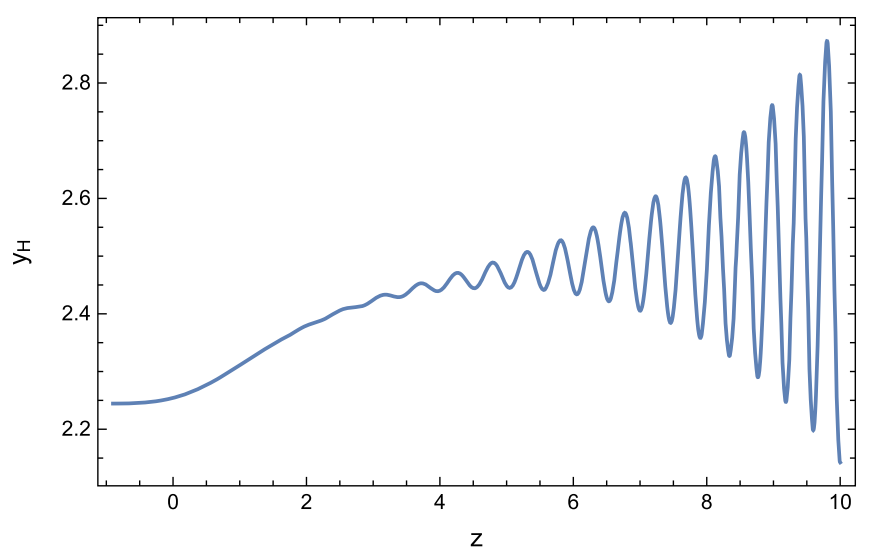

Before closing, let us briefly discuss the stability of the cosmological perturbations for the $R+f(\mathcal{G})$ gravity as the general-relativistic limit is approached. This issue was covered in detail in Ref. [65], so we only report their result which is that the condition $f^{\prime \prime}(\mathcal{G})>0$ suffices to ensure the stability of any solution in the general-relativistic limit. In our case, during the late-time era, where $f(\mathcal{G}) \sim c_{1} / \mathcal{G}$, the quantity $f^{\prime \prime}(\mathcal{G})$ is equal to $f^{\prime \prime}((\mathcal{G}))=\frac{2 c_{1}}{\mathcal{G}^{3}}$, and hence stability is ensured. However, for the early-time era, in which case the $f(\mathcal{G})$ gravity has the form $f(\mathcal{G}) \sim c_{2} \mathcal{G}^{\frac{\alpha}{3 \alpha-1}}$, the quantity $f^{\prime \prime}(\mathcal{G})$ reads

$$
f^{\prime \prime}(\mathcal{G})=-\frac{\alpha(2 \alpha-1) c_{2} \mathcal{G}^{\frac{\alpha}{3 \alpha-1}-2}}{(1-3 \alpha)^{2}}
$$

which is obviously negative for the values of $\alpha$ we chose, and hence this solution is unstable in the general-relativistic limit. However, this is not a problem in this case, because near the bounce era no consistent general-relativistic limit exists, since general relativity cannot describe the early-time era, while during the late times there can be some overlap between the general-relativistic description and the $f(\mathcal{G})$ description, because the late-time era is approximately described by a nearly de Sitter evolution.

\section{GENERALIZED $f(R, \mathcal{G})$ GRAVITY LATE-TIME PHENOMENOLOGY: THE CASE OF $f(R)+g(\mathcal{G})$ GRAVITY}

In the previous section we compared the results of the Gauss-Bonnet late-time phenomenology with the $f(R)$ model of Eq. (48), and we demonstrated that even though both the $f(R)$ and $R+f(\mathcal{G})$ models are capable of uniting early- and late-time eras there exist many differences, in particular the dark energy oscillations spotted at redshifts $z \geq 4$, which are present in the $f(R)$ gravity model but absent in the Gauss-Bonnet model. It is therefore sensible

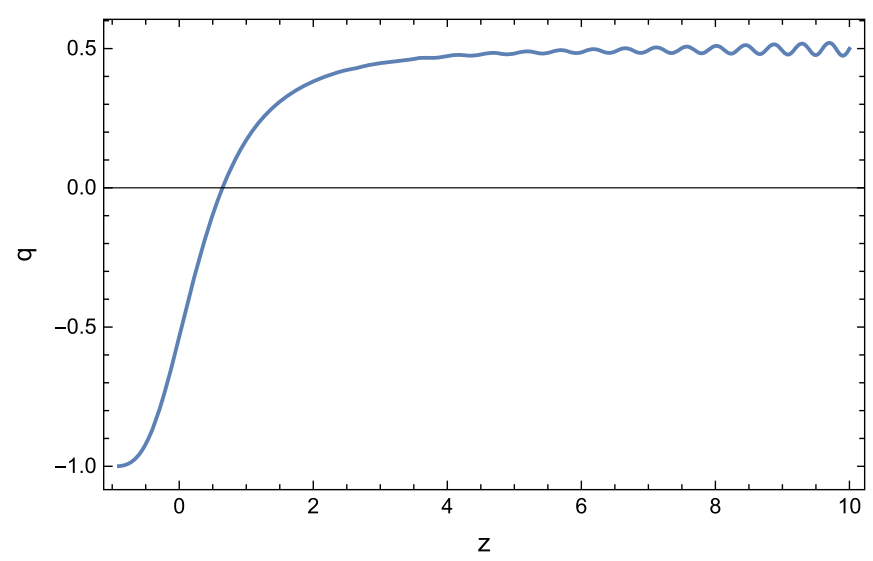

FIG. 6. Functions $y_{H}(z)$ (left) and $q(z)$ (right) for the $f(R)+g(\mathcal{G})$ model with the exact same initial conditions as in the previous section. In this case, we clearly see that dark energy oscillations have not been nullified and, as a matter of fact, the same behavior as in the pure $f(R)$ case is produced. The only difference lies in the numerical values of the statefinders, which will be addressed shortly. 

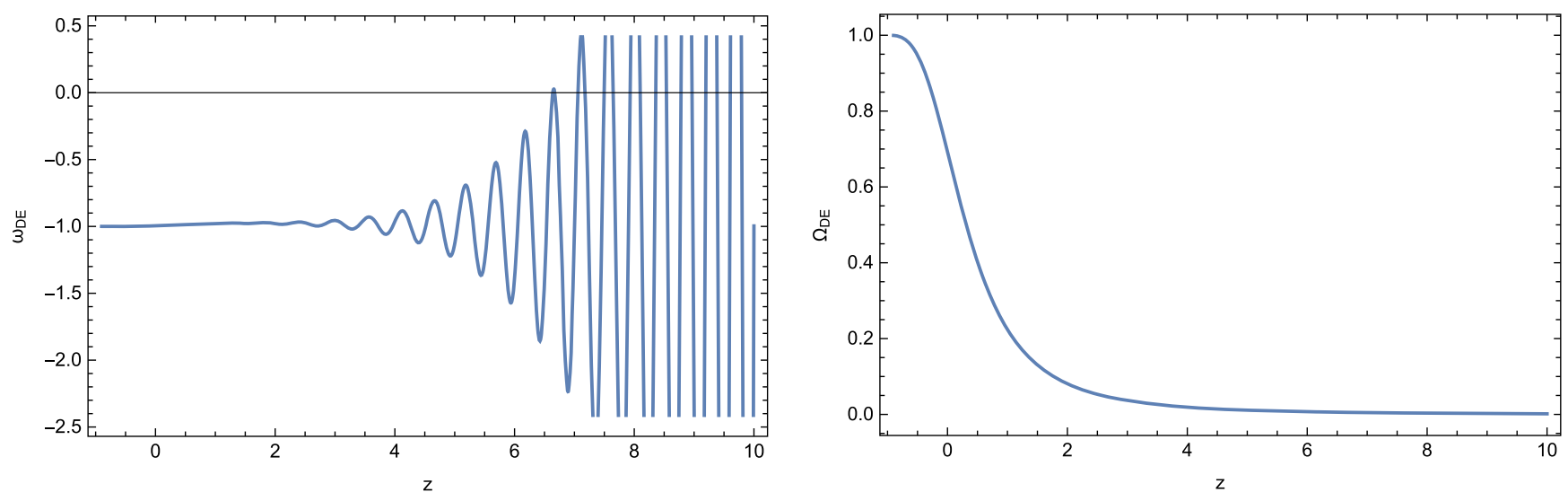

FIG. 7. Dark energy EoS parameter $\omega_{\mathrm{DE}}$ (left) and dark energy density parameter $\Omega_{\mathrm{DE}}$ (right). As in the pure $f(R)$ case, only the EoS parameter appears to oscillate for large redshifts.

to try to find out whether combining the aforementioned $f(R)$ model with an appropriately chosen $g(\mathcal{G})$ function makes the dark energy oscillations disappear. Therefore, let us assume that the $f(R, \mathcal{G})$ model is written as $f(R, \mathcal{G})=f(R)+g(\mathcal{G})$, with

$$
f(R)=R+\left(\frac{R}{M}\right)^{2}-\gamma \Lambda\left(\frac{R}{3 m_{s}^{2}}\right)^{\delta}
$$
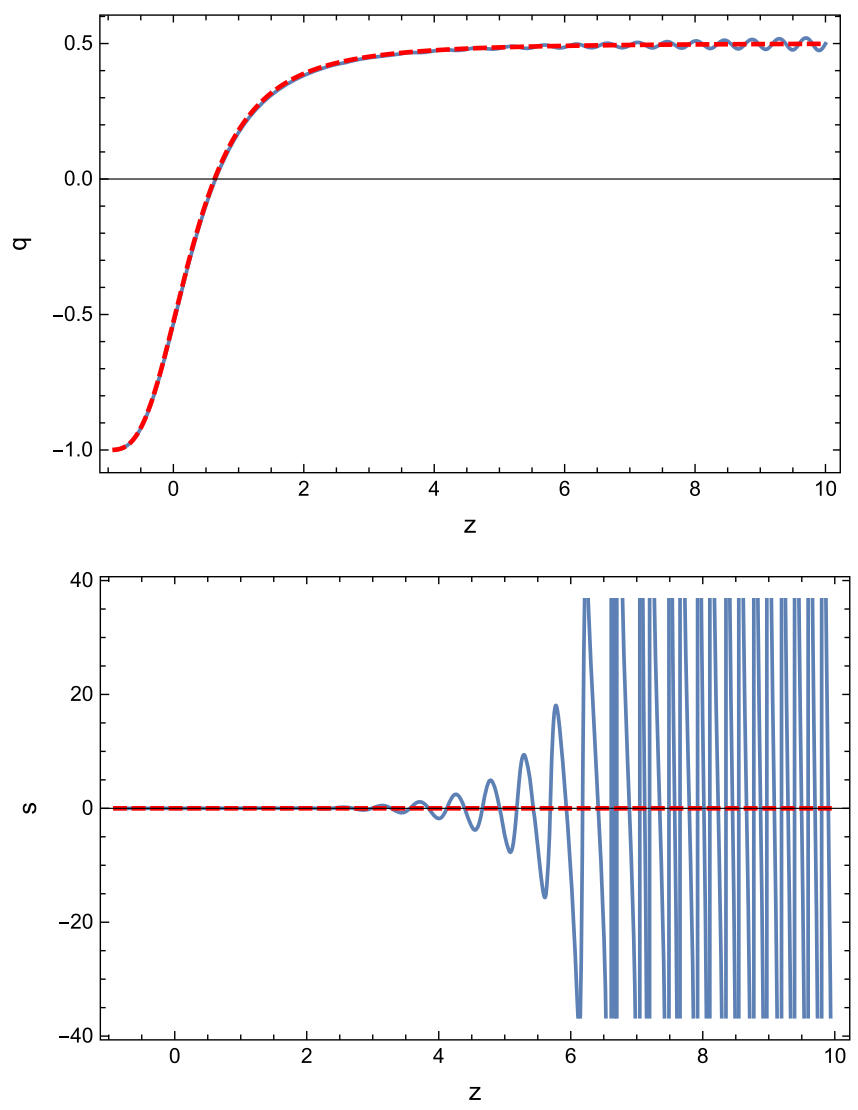

and

$$
g(\mathcal{G})=\frac{\lambda}{\sqrt{\beta}} \mathcal{G} \tan ^{-1}\left(\frac{\mathcal{G}}{\beta}\right)
$$

where $M, \gamma$, and $\delta$ are the same parameters as in the previous section, whereas $\lambda$ and $\beta$ are extra auxiliary parameters, with the first being dimensionless and the latter having mass dimension $[m]^{4}$ for consistency, and
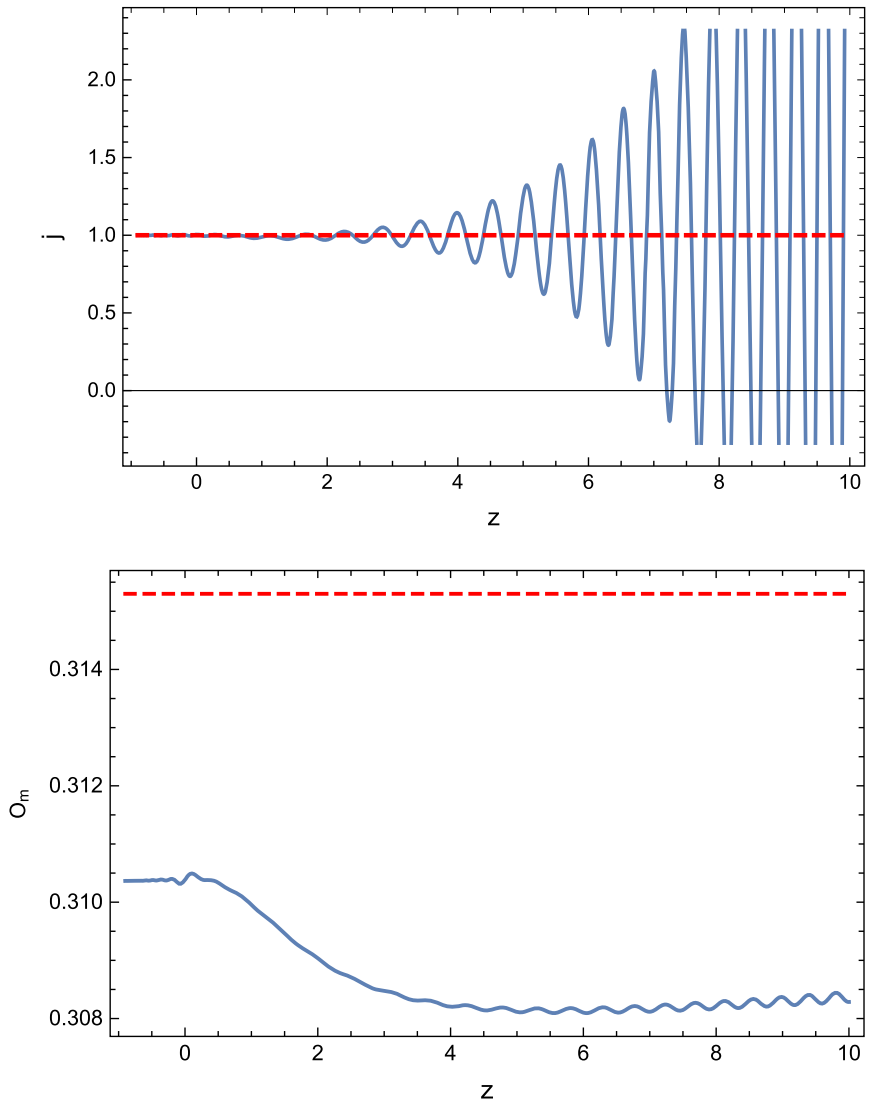

FIG. 8. Direct comparison of several statefinder quantities between the $f(R)+g(\mathcal{G})$ model and $\Lambda$ CDM. 
moreover $\beta$ is assumed to be positive. The model $g(\mathcal{G})$ seems quite convenient for our approach since it has a linear part in terms of $\mathcal{G}$, and moreover the derivative of $\tan ^{-1}(\mathcal{G})$ produces a term $\frac{1}{1+\mathcal{G}^{2}}$. Since we assume an $f(R)+$ $g(\mathcal{G})$ gravity, Eq. (5) is written as follows:

$3 f_{R} H^{2}=\kappa^{2} \rho_{(m)}+\frac{R f_{R}-f}{2}-3 H \dot{f}_{R}+\frac{\mathcal{G} g_{\mathcal{G}}-g}{2}-12 \dot{g}_{\mathcal{G}} H^{3}$.

This is the Friedmann equation, and this particular differential equation will be solved numerically in the same redshift interval $z=[-0.9,10]$ by expressing the above differential equation in terms of the statefinder quantity $y_{H}$. In the case at hand, we have

$$
\begin{gathered}
f_{R}=1+\frac{2 R}{M^{2}}-\frac{\gamma \delta \Lambda}{\left(3 m_{s}^{2}\right)^{\delta}} R^{\delta-1}, \\
\dot{f}_{R}=\dot{R}\left(\frac{2}{M^{2}}-\frac{\gamma \delta(1-\delta) \Lambda}{\left(3 m_{s}^{2}\right)^{\delta}} R^{\delta-2}\right), \\
g_{\mathcal{G}}=\frac{\lambda}{\sqrt{\beta}}\left(\tan ^{-1}\left(\frac{\mathcal{G}}{\beta}\right)+\frac{\beta \mathcal{G}}{\beta^{2}+\mathcal{G}^{2}}\right), \\
\dot{g}_{\mathcal{G}}=2 \sqrt{\beta} \lambda \dot{\mathcal{G}} \frac{\mathcal{G}}{\beta^{2}+\mathcal{G}^{2}}\left(1-\frac{\mathcal{G}}{\beta^{2}+\mathcal{G}^{2}}\right) .
\end{gathered}
$$

Suppose now that the values of $M, m_{s}, \gamma, \delta$, and $\Lambda$ remain the same as in the previous section (hence the reason that they were not relabeled), and in addition $\lambda=1, \beta=1$. Then, we use exactly the same initial conditions to numerically solve the differential equation (70), and the results of our analysis are presented in Figs. 6, 7, and 8.

In particular, in Fig. 6 we plot the deceleration parameter and statefinder $y_{H}$ as functions of the redshift, and in Fig. 7 we plot the dark energy EoS parameter and dark energy density parameter as functions of the redshift. Finally, in Fig. 8 we plot several statefinders for the combined $f(R)+$ $g(\mathcal{G})$ model (blue curves) and we compare these to the $\Lambda \mathrm{CDM}$ model results (red curves). Also, in Table II we compare the values of several quantities of cosmological interest at present time for the combined $f(R)+g(\mathcal{G})$ model, and compare these to the pure $f(R)$ gravity model. An overall apparent result derived from the analysis is that the presence of the $f(R)$ gravity affects the late-time phenomenology, since it brings along the dark energy oscillations in several statefinder quantities and cosmological quantities. This result seems to be model independent
TABLE II. Comparison between the values of the pure $f(R)$ model and the $f(R)+g(\mathcal{G})$ case. Even though all of the statefinders seem to have the same behavior in the redshift interval $z=[-0.9,10]$, the current values are indeed different, however to a small extent.

\begin{tabular}{lcc}
\hline \hline Parameter & $f(R)$ Value & $f(R)+g(\mathcal{G})$ Value \\
\hline$q(z=0)$ & -0.520954 & -0.53442 \\
$j(z=0)$ & 1.00319 & 1.00478 \\
$s(z=0)$ & -0.00104169 & -0.00154146 \\
$O m(z=0)$ & 0.319364 & 0.310387 \\
$\Omega_{\mathrm{DE}}(0)$ & 0.683948 & 0.691643 \\
$\omega_{\mathrm{DE}}(0)$ & -0.995205 & -0.995673 \\
\hline \hline
\end{tabular}

and thus, in conclusion, the $f(R)$ gravity dark energy oscillations cannot be remedied by adding a GaussBonnet term to the Lagrangian.

\section{CONCLUSIONS}

In this paper we quantitatively addressed the latetime phenomenology of Gauss-Bonnet theories, and we investigated how a singular primordial bouncing cosmology and a viable dark energy era can be realized by GaussBonnet gravity. We were able to realize this unification scheme by using a Gauss-Bonnet gravity of the form $R+f(\mathcal{G})$, which is free from primordial superluminal modes. The type IV singular bounce at early times, realized by an appropriate Gauss-Bonnet gravity, generates a nearly scale-invariant power spectrum of the primordial scalar curvature perturbations. In addition, the late-time driving part of the $R+f(\mathcal{G})$ gravity generates a viable dark energy era, which mimics the $\Lambda$ CDM model for some statefinder quantities, and is compatible at present time with the latest Planck data on cosmological parameters. This specific model has another appealing attribute: the dark energy era is free from large-redshift $(z \sim 4)$ dark energy oscillations, which are known to occur in $f(R)$ theories. For demonstrative purposes, we compared the $R+f(\mathcal{G})$ model with the $\Lambda \mathrm{CDM}$ model and an appropriate $f(R)$ gravity model that is known to generate a viable dark energy, and we provided qualitative evidence for the presence of dark energy oscillations only in the $f(R)$ gravity case. In order to further analyze the impact of a nontrivial $f(R)$ gravity term in the dark energy oscillations, we also studied the late-time phenomenology of an $f(R)+g(\mathcal{G})$ model, and showed that the dark energy oscillations are also present in this case. Thus, the $f(R)$ gravity part of the gravitational action seems to always lead to dark energy oscillations at large redshifts. 
[1] G. Bertone, D. Hooper, and J. Silk, Phys. Rep. 405, 279 (2005).

[2] L. Bergstrom, Rep. Prog. Phys. 63, 793 (2000).

[3] Y. Mambrini, S. Profumo, and F. S. Queiroz, Phys. Lett. B 760, 807 (2016).

[4] S. Profumo, arXiv:1301.0952.

[5] D. Hooper and S. Profumo, Phys. Rep. 453, 29 (2007).

[6] V. K. Oikonomou, J. D. Vergados, and C. C. Moustakidis, Nucl. Phys. B773, 19 (2007).

[7] S. Capozziello and M. De Laurentis, Ann. Phys. (Amsterdam) 524, 545 (2012).

[8] A. G. Riess et al. (Supernova Search Team), Astron. J. 116, 1009 (1998).

[9] K. Bamba, S. Capozziello, S. Nojiri, and S. D. Odintsov, Astrophys. Space Sci. 342, 155 (2012).

[10] P. J. E. Peebles and B. Ratra, Rev. Mod. Phys. 75, 559 (2003).

[11] M. Li, X. D. Li, S. Wang, and Y. Wang, Commun. Theor. Phys. 56, 525 (2011).

[12] K. Bamba, C. Q. Geng, C. C. Lee, and L. W. Luo, J. Cosmol. Astropart. Phys. 01 (2011) 021.

[13] J. Frieman, M. Turner, and D. Huterer, Annu. Rev. Astron. Astrophys. 46, 385 (2008).

[14] C. G. Boehmer, G. Caldera-Cabral, R. Lazkoz, and R. Maartens, Phys. Rev. D 78, 023505 (2008).

[15] S. Nojiri and S. D. Odintsov, Phys. Rev. D 74, 086005 (2006).

[16] E. Elizalde, S. Nojiri, and S. D. Odintsov, Phys. Rev. D 70, 043539 (2004).

[17] A. N. Makarenko and A. N. Myagky, Int. J. Geom. Methods Mod. Phys. 15, 1850096 (2018).

[18] S. Capozziello, V. F. Cardone, S. Carloni, and A. Troisi, Int. J. Mod. Phys. D 12, 1969 (2003).

[19] A. Y. Kamenshchik, U. Moschella, and V. Pasquier, Phys. Lett. B 511, 265 (2001).

[20] S. M. Carroll, Phys. Rev. Lett. 81, 3067 (1998).

[21] S. Capozziello, Int. J. Mod. Phys. D 11, 483 (2002).

[22] S. Capozziello, V. F. Cardone, E. Piedipalumbo, and C. Rubano, Classical Quantum Gravity 23, 1205 (2006).

[23] S. Nojiri, S. D. Odintsov, and V. K. Oikonomou, Phys. Rep. 692, 1 (2017).

[24] S. Nojiri and S. D. Odintsov, Phys. Rep. 505, 59 (2011).

[25] S. Nojiri and S. D. Odintsov, eConf C0602061, 06 (2006); Int. J. Geom. Methods Mod. Phys. 04, 115 (2007).

[26] S. Capozziello and M. De Laurentis, Phys. Rep. 509, 167 (2011); V. Faraoni and S. Capozziello, Fundam. Theor. Phys. 170 (2010).

[27] A. de la Cruz-Dombriz and D. Saez-Gomez, Entropy 14, 1717 (2012).

[28] G. J. Olmo, Int. J. Mod. Phys. D 20, 413 (2011).

[29] A. H. Guth, Phys. Rev. D 23, 347 (1981).

[30] A. D. Linde, Phys. Rev. D 49, 748 (1994).

[31] A. D. Linde, Phys. Lett. 129B, 177 (1983).

[32] R. Brandenberger and P. Peter, Found. Phys. 47, 797 (2017).

[33] J. de Haro and Y. F. Cai, Gen. Relativ. Gravit. 47, 95 (2015).

[34] Y. F. Cai, Sci. China Phys. Mech. Astron. 57, 1414 (2014).

[35] P. P. Avelino and R. Z. Ferreira, Phys. Rev. D 86, 041501 (2012).

[36] M. Koehn, J. L. Lehners, and B. A. Ovrut, Phys. Rev. D 90, 025005 (2014).
[37] Y.F. Cai, E. McDonough, F. Duplessis, and R. H. Brandenberger, J. Cosmol. Astropart. Phys. 10 (2013) 024.

[38] R. H. Brandenberger, arXiv:1206.4196.

[39] Y.F. Cai, R. Brandenberger, and X. Zhang, J. Cosmol. Astropart. Phys. 03 (2011) 003.

[40] L. E. Allen and D. Wands, Phys. Rev. D 70, 063515 (2004).

[41] S. Nojiri and S. D. Odintsov, Phys. Rev. D 68, 123512 (2003).

[42] S. D. Odintsov and V. K. Oikonomou, Phys. Rev. D 101, 044009 (2020).

[43] R. R. Caldwell, M. Kamionkowski, and N. N. Weinberg, Phys. Rev. Lett. 91, 071301 (2003).

[44] B. Li, J. D. Barrow, and D. F. Mota, Phys. Rev. D 76, 044027 (2007).

[45] S. Nojiri and S. D. Odintsov, Phys. Lett. B 631, 1 (2005).

[46] E. Elizalde, S. D. Odintsov, V. K. Oikonomou, and T. Paul, Nucl. Phys. B954, 114984 (2020).

[47] G. Cognola, E. Elizalde, S. Nojiri, S. D. Odintsov, and S. Zerbini, Phys. Rev. D 73, 084007 (2006).

[48] E. Elizalde, R. Myrzakulov, V. V. Obukhov, and D. SaezGomez, Classical Quantum Gravity 27, 095007 (2010).

[49] K. Izumi, Phys. Rev. D 90, 044037 (2014).

[50] V. K. Oikonomou, Astrophys. Space Sci. 361, 211 (2016).

[51] K. Kleidis and V. K. Oikonomou, Int. J. Geom. Methods Mod. Phys. 15, 1850064 (2018).

[52] V. K. Oikonomou, Phys. Rev. D 92, 124027 (2015).

[53] A. Escofet and E. Elizalde, Mod. Phys. Lett. A 31, 1650108 (2016).

[54] K. Bamba, A. N. Makarenko, A. N. Myagky, and S. D. Odintsov, Phys. Lett. B 732, 349 (2014).

[55] A. N. Makarenko, Int. J. Geom. Methods Mod. Phys. 13, 1630006 (2016).

[56] G. Navo and E. Elizalde, Int. J. Geom. Methods Mod. Phys. 17, 2050162 (2020).

[57] F. Bajardi and S. Capozziello, Eur. Phys. J. C 80, 704 (2020).

[58] S. Capozziello, C. A. Mantica, and L. G. Molinari, Int. J. Geom. Methods Mod. Phys. 16, 1950133 (2019).

[59] M. Benetti, S. Santos da Costa, S. Capozziello, J. S. Alcaniz, and M. De Laurentis, Int. J. Mod. Phys. D 27, 1850084 (2018).

[60] T. Clifton and J. D. Barrow, Classical Quantum Gravity 23, 2951 (2006).

[61] C. Bogdanos, S. Capozziello, M. De Laurentis, and S. Nesseris, Astropart. Phys. 34, 236 (2010).

[62] S. Capozziello, V. F. Cardone, S. Carloni, and A. Troisi, Phys. Lett. A 326, 292 (2004).

[63] K. Bamba, S. D. Odintsov, L. Sebastiani, and S. Zerbini, Eur. Phys. J. C 67, 295 (2010).

[64] M. De Laurentis, M. Paolella, and S. Capozziello, Phys. Rev. D 91, 083531 (2015).

[65] A. de la Cruz-Dombriz and D. Saez-Gomez, Classical Quantum Gravity 29, 245014 (2012).

[66] N. Aghanim et al. (Planck Collaboration), Astron. Astrophys. 641, A6 (2020).

[67] Y. Akrami et al. (Planck Collaboration), Astrophys. Space Sci. 364, 69 (2019).

[68] K. Bamba, A. N. Makarenko, A. N. Myagky, and S. D. Odintsov, Phys. Lett. B 732, 349 (2014). 